Pacific Journal of Mathematics

HOLOMORPHIC FUNCTIONAL AND COMPLEX CONVEXITY 


\title{
HOLOMORPHIC FUNCTIONALS AND COMPLEX CONVEXITY IN BANACH SPACES
}

\author{
H. J. BREMERMANN
}

1. Introduction. The present paper extends some basic theorems of the theory of several complex variables to Banach spaces. Results which are new even for finite dimension are also obtained. Considerable use is made of methods developed in "Complex Convexity" (Bremermann [8]), however, many modifications are necessary to adapt them to infinite dimension.

A complex valued functional is Gâteaux holomorphic (or in short Gholomorphic) in a domain $D$ of a complex Banach space $B_{c}$ if it is single valued and its restriction to an arbitrary analytic plane $\left\{z \mid z=z_{0}+\lambda a\right\}$ ( $z_{0} \in D, a \in B_{c}, \lambda$ a complex parameter) is a holomorphic function of $\lambda$ in the intersection of the plane with $D$. The space of $n$ complex variables $C^{n}$ can be considered as a Banach space, and for $C^{n}$ the above definition is equivalent to the usual definition of a holomorphic function of several complex variables. In an infinite dimensional Banach space the Gâteaux holomorphic functions are not necessarily locally bounded, while in a finite dimensional space the local boundedness is a consequence of holomorphy. Therefore another notion of holomorphy, also coinciding with the notion of holomorphy in finite dimensional spaces, is possible: A function is Fréchet holomorphic in a domain $D$ if it is Gâteaux holomorphic and locally bounded (compare Hille [11] and Soeder [17]). The theories of both types of holomorphic functions have been studied, the latter more than the former. Both theories are considerably less developed than the theory of finitely many variables. This may be partly due to the fact that the infinite dimensional spaces are not locally compact, in fact, if a space is locally compact, then it is finite dimensional (see Hille [11]).

In the present paper the theory of Gâteaux holomorphic functionals is studied exclusively. As a tool are used plurisubharmonic functionals (as defined by Oka [14] and [15], Lelong [12] and Thorin [19]) and a functional $d_{D}^{(N)}(z)$ which is the distance of the point $z$ from the boundary of the domain $D$ measured in the norm $N$. A notion of holomorphic continuation is defined and a "basic lemma" on the simultaneous continuation of $G$-holomorphic functionals is proved (3.1). ${ }^{1}$ A consequence of

Received April 26, 1956. This research was supported by the United States Air Force, through the Office of Scientific Recearch of the Air Research and Development Command.

1 The lemma in its present form is new also for finite dimension and permits to construct the envelope of an arbitrary domain in the $C^{n}$ explicitely. This will be carried out in a further paper. 
this lemma is the fact that there exist domains, as in finite dimension, such that all $G$-holomorphic functions can be continued $G$-holomorphically into a larger domain. Those domains for which a $G$-holomorphic function exists that is not continuable, are called domains of holomorphy. From the continuation lemma follows that the domains of holomorphy have the property that the functional $-\log d_{D}^{(N)}(z)$ is plurisubharmonic in $D$, and a theorem is proved which for finite dimension is known as "Kontinuitätssatz." The property of the functional $-\log d_{D}^{(N)}(z)$ to be plurisubharmonic is invariant with respect to all norms $N$ that generate equivalent topologies. The domains for which $-\log d_{D}^{(N)}(z)$ is plurisubharmonic are called pseudo-convex, and some of their properties are studied. A domain $D$ in a complex Banach space $B_{c}$ is pseudo-convex if and only if its intersection with every (complex) two-dimensional linear submanifold of $B$ is pseudo-convex.

The notion of pseudo-convexity bears some formal relationship to the ordinary convexity in real spaces, this is established by showing: A domain $D$ in a real Banach $B_{r}$ is convex if and only if $-\log d_{D}^{(N)}(x)$ is a convex functional in $D$. Finally tube domains are studied, that is domains of the form $\left\{z \mid x \in d_{x}, y\right.$ arbitrary $\}$, where $x$ is the real part and $y$ the imaginary part of $z$, and $d_{x}$ a domain in the real Banach space of the real parts. It is shown that for this particular class of domains the two notions coincide: A tube domain is pseudo-convex if and only if it is convex.

For simplicity's sake the present considerations are limited to complex valued functionals but can be extended without difficulty to vector valued functions. Also generalizations to spaces more general than Banach spaces (for instance locally convex spaces) are possible.

\section{Holomorphic, plurisubharmonic and distance functionals.}

2.1. We will consider in this paper Banach spaces where the field of scalars is either the field of real numbers or the field of complex numbers. Accordingly we speak of real and complex Banach spaces and write $B_{r}$ and $B_{c}$ respectively. By $z$ we will denote exclusively elements of complex $B$-spaces and by $x$ elements of real $B$-spaces.

2.2. The norm that is defined in a Banach space $B$ provides it in a natural way with a topology (strong topology). As neighborhoods of a point $a \in B$ we define the pointsets $\{b \mid\|b-a\|<\varepsilon\}$. A region is an open set; a domain is an open and connected set.

2.3. Definition. Let $\lambda$ be a complex parameter. A complex valued functional $f(z)$, defined in a domain $D$ of a complex Banach space $B_{c}$ is 
Gâteaux holomorphic in $D$ (or in short $G$-holomorphic) if $f(z)$ is singlevalued in $D$ and if $f\left(z_{0}+\lambda a\right)$ is holomorphic in $\lambda$ at the point $\lambda=0$ for all $z_{0} \in D$ and $a \in B$. In other words $f(z)$ is required to be a holomorphic function of the one complex variable $\lambda$ on the intersection of any twodimensional analytic plane $\left\{z \mid z=z_{0}+\lambda a\right\}$ with the domain $D$. Obviously a function is $G$-holomorphic in $D$ if and only if it is single-valued and $G$-holomorphic in a neighborhood of each point of $D$ (locally holomorphic). This definition is equivalent to the requirement that the Gâteaux differential exists everywhere in $D$ and that $f(z)$ is single-valued in $D$. We do not require that $f(z)$ be locally bounded or similar conditions. (Compare Hille [11], p. 71 and p. 81).

2.4. Definition. A real-valued functional $V(z)$ defined in a domain $D$ of a complex Banach space $B_{c}$ is quasi-plurisubharmonic in $D$ if $V\left(z_{0}+\lambda a\right)$ is quasi-subharmonic in $\lambda$ at the point $\lambda=0$ for all $z_{0} \in D$ and $a \in B_{c} . \quad V(z)$ is plurisubharmonic if $V(z)$ is quasi-plurisubharmonic and upper-semicontinuous in $D$. (Cf. Thorin [19], p. 16) $V(z)$ is uppersemicontinuous at the point $z_{0}$ if for every $\varepsilon>0$ there exists a $\delta$, such that $V(z)-V\left(z_{0}\right)<\varepsilon$ for $\left\|z-z_{0}\right\|<\delta$. (For the definition of quasi-plurisubharmonic see T. Radó [16]. Cf. also P. Lelong [12]). What we call quasi-plurisubharmonic functions Lelong denotes as functions of class $M$. The plurisubharmonic functions have also been introduced by $\mathrm{K}$. Oka [14] and [15] under the name pseudo-convex functions. Oka admits the constant, $-\infty$, Lelong excludes it. For our applications it is more convenient to admit $-\infty$ as a plurisubharmonic functional.)

2.5. We now have to define the notion of holomorphic continuation. In one and several variables this is being done by means of power series developments. However, power series are somewhat inconvenient here. Therefore we will define as holomorphic continuation a function that is holomorphic in a larger domain and coincides with the given function in the given domain. However, already in one variable the "larger domains" may be no longer schlicht but concrete complex manifolds with no branch points as interior points. We have to take care of this situation and therefore define the following.

2.6. $D$ is a domain over the space $B_{c}$ if $D$ is a topological space carrying a mapping $\varphi_{D}$ which maps $D$ into $B_{c}$, such that $\varphi_{D}$ is locally a homeomorphism.

We call $\varphi_{D}$ the projection mapping of $D$ and $\varphi_{D}(E)$, where $E$ is a set in $D$, the projection of $E$.

Domains over a space $B_{c}$ are special complex analytic manifolds of infinite dimension. (For general complex analytic manifolds of infinite 
dimension, ef. J. Eells [10].)

2.7. $D$ is a continuation of a domain $D_{0}$ over $B_{c}$ if there exists a subset $\tilde{D}_{0}$ of $D$ and a homeomorphism $h$ of $\tilde{D}_{0}$ onto $D_{0}$ such that $\varphi_{D_{0}} h(P)=$ $\varphi_{D}(P)$ for every $P$ in $\tilde{D_{0}}$.

We can then identify $\tilde{D}_{0}$ and $D_{0}$. In particular if $D_{0}$ is a domain in $B_{c}$-we will also say schlicht domain - then $D$ is a continuation of $D_{0}$ if there exists a subset $\tilde{D}_{0} \subset D$ such that $\varphi_{D}\left(\tilde{D_{0}}\right)$ is a homeomorphism onto $D_{0}$.

2.8. A functional $f$ is $G$-holomorphic in a domain $D$ over a complex Banach space $B_{c}$ if $f$ is $G$-holomorphic in a neighborhood of each point in $D$. And it is $G$-holomorphic in a neighborhood $U_{i}$ if it is $G$-holomorphic in the homeomorphic image $\varphi_{D} U_{i}$ which is an open set in $B_{c}$ where the notion of $G$-holomorphy is defined (2.3).

2.9. Let $f(z)$ be a $G$-holomorphic functional in a domain $D \subset B$. Then $g(z)$ is a $G$-holomorphic continuation of $f(z)$ if $g(z)$ is $G$-holomorphic in a continuation $D_{1}$ of $D$ and coincides with $f(z)$ in $D$.

2.10. Uniqueness of the G-holomorphic continuation. Let $D$ be a domain over $B_{c}$. Let $D^{*}$ be a subdomain. Let $f(z)$ and $g(z)$ be $G$ holomorphic functionals in $D$, let $f(z) \equiv g(z)$ in $D^{*}$, then we have $f(z) \equiv$ $g(z)$ throughout $D$.

Proof. Let $S$ be the set of points such that $f(z)$ and $g(z)$ coincide. Then we have $D^{*} \subset S \subset D$. Let $S^{*}$ be the largest open set contained in $S$. Suppose $S^{*} \neq D$. Then there exists a boundary point $z_{0}$ of $S^{*}$ which is an interior point of $D$. Let $U$ be the homeomorphic image of a neighborhood of $z_{0}$ in the $B_{c}$. In particular we can choose $U$ as a sphere. In this sphere we have a point $z_{1}$ such that in a neighborhood of $z_{1}$ we have $f(z)=g(z)$ and a point $z_{2}$ such that $f\left(z_{2}\right) \neq g\left(z_{2}\right)$. Then we connect $z_{1}$ and $z_{2}$ by an analytic plane which cuts $U$ in a circle. Restricting $f(z)$ and $g(z)$ to the analytic plane we obtain a contradiction to the identity theorem of holomorphic functions in one variable.

2.11. A domain $H$ for which a functional $f(z)$ exists that is $G$ holomorphic in $H$ and does not possess a $G$-holomorphic continuation into a proper continuation of $H$ we call a domain of holomorphy.

2.12. The distance function. Let $D$ be a domain in $B$, then we associate with every point of $D$ the value 


$$
d_{D}^{(N)}(z)=\sup r \ni\left\{z^{\prime} \mid\left\|z^{\prime}-z\right\|<r\right\} \subset D,
$$

in other words $d_{D}^{(N)}(z)$ is the distance of the point $z$ from the boundary of $D$ measured in the norm $N$.

If $D$ is different from the whole space $B$, then $D$ has at least one finite boundary point, and then obviously $d_{D}^{(N)}(z)$ is finite in $D$. If $D$ is the whole space $B_{c}$, then $d_{D}^{(N)} \equiv \infty$.

2.13. If $D$ is different from the whole space, then $d_{D}^{(N)}(z)$ is continuous with respect to the topology generated by the norm $N$.

The poof is the same as in the finite case which is carried out in Bremermann [8].

2.14. Definition. Besides the distance function $d_{D}^{(N)}(z)$ we will consider the distance function

$$
d_{a, D}^{(N)}(z)=\sup r \ni\left\{z^{\prime}\left|z^{\prime}=z+\lambda a,\|a\|_{N}=1,\right| \lambda \mid<r\right\} \subset D,
$$

in other words $d_{a, D}^{(N)}(z)$ is the radius of the largest circle with center at $z$ on the analytic plane $\left\{z^{\prime} \mid z^{\prime}=z+\lambda a\right\}$ that is contained in $D$, that is the distance of $z$ from the boundary of $\left\{z^{\prime} \mid z^{\prime}=z+\lambda a\right\} \cap D$.

From the definitions it follows immediately the relation

$$
d_{D}^{(N)}(z)=\inf _{a}\left\{d_{a, D}^{(N)}(z)\right\}
$$

where $a$ varies through all elements of $B$ with norm 1 .

2.15. The function $d_{a, D}^{(N)}(z)$ is lower semicontinuous with respect to the topology generated by the norm $N$.

If $D$ is the whole space, then $d_{a, D}^{(N)}(z)$ will be $\equiv \infty$. However, even if $D$ is not the whole space, $d_{a, D}^{(N)}(z)$ can be infinite for certain directions, though not for all directions.

(1) Let $d_{a, D}^{(N)}\left(z_{1}\right)=c_{1}$ be finite. Then for every $\varepsilon>0$ the point set

$$
\left\{z\left|z=z_{1}+\lambda a,\right| \lambda \mid \leqq c_{1}-\varepsilon\right\}
$$

is compact in $D$. Hence there exists for every $\varepsilon>0$ a $\delta$ such that for $\left\|z_{1}-z_{2}\right\|<\delta$ the point set

$$
\left\{z\left|z=z_{2}+\lambda a,\right| \lambda \mid \leqq c_{1}-\varepsilon\right\}
$$

is contained in $D$. Hence for $\left\|z_{1}-z_{2}\right\|<\delta$ we have

$$
\begin{aligned}
& d_{a, D}^{(N)}\left(z_{2}\right)>c_{1}-\varepsilon, \quad \text { or } \\
& d_{a, D}^{(N)}\left(z_{1}\right)-d_{a, D}^{(N)}\left(z_{2}\right)<\varepsilon .
\end{aligned}
$$

Hence $d_{a, D}^{(N)}(z)$ is lower semicontinuous at $z_{1}$ with respect to the norm $N$. 
(2) Let $d_{a, D}^{(N)}\left(z_{1}\right)=\infty$. Then for arbitrary large $M$ the point set

$$
\left\{z\left|z=z_{1}+\lambda \alpha,\right| \lambda \mid \leqq M\right\}
$$

is compact in $D$. Hence there exists a $\delta$ such that for $\left\|z_{1}-z_{2}\right\|<\delta$ the point set

$$
\left\{z\left|z=z_{2}+\lambda a,\right| \lambda \mid \leqq M\right\}
$$

is contained in $D$. Hence

$$
d_{a, D}^{(N)}\left(z_{2}\right)>M \text { for }\left\|z_{1}-z_{2}\right\|<\delta .
$$

That means that also in this case $d_{a, D}^{(N)}(z)$ is lower semicontinuous at the point $z_{1}$ with respect to the norm $N$.

2.16. By a similar argument it follows that $d_{a, D}^{(N)}(z)$ is for fixed $z$ lower semicontinuous with respect to variable direction $a$.

\section{Simultaneous holomorphic continuation.}

3.1. Fundamental lemma. Let $D$ be a domain in a complex Banach space. Let $S$ be a simply connected domain on an analytic plane $\left\{z \mid z=z_{0}+\lambda b\right\}$. Let $T$ be the boundary of $S$ and let $S \cup T \subset D$. Let $X(\lambda)$ be a function holomorphic in the image of $S$ in the $\lambda$-parameter planein the following we will simply say holomorphic in $S$-and let $X(\lambda) \neq 0$ in $S \cup T$ and $|X(\lambda)|$ continuous in $S \cup T$.

Let

$$
|X(\lambda)| d_{a, D}^{(N)}\left(z_{0}+\lambda b\right) \geqq m>0 \quad \text { for } \lambda \in T .
$$

Then any functional that is G-holomorphic in $D$ can be continued G-holomorphically into all points

$$
C=\left\{\left.z\left|z=z_{0}+\lambda b+\tau a, \quad \lambda \in S \cup T,\right| \tau|<m| X(\lambda)\right|^{-1}\right\},
$$

( $\tau$ a complex parameter).

The idea of the proof is the following. We consider the subspace $\left\{z \mid z=z_{0}+\lambda b+\tau a\right\}$ and an arbitrary functional $f(z)$. The restriction of $f$ to the intersection of $D$ with this subspace is a holomorphic function in $\lambda$ and $\tau$. For fixed $\lambda$ we can develop $f\left(z_{0}+\lambda b+\tau a\right)$ into an ordinary power series of powers of $\tau$. From the maximum principle we derive that this series converges in the pointset $C$. Thus we have continued $f$ into $C$. However it has to be checked that the continuation is not only a continuation of $f$ as a holomorphic function of the one variable $\tau$ but as a $G$-holomorphic function in $B_{c}$.

This is not trivial. Functions of two complex variables are known 
which can be continued as functions of one variable beyond the domain where they are holomorphic in both variables. (Cf. Behnke-Thullen [3].)

In order to show that $f(z)$ is holomorphic in an arbitrary point $P$ of $C$ it is sufficient to show that $f(z)$ can be defined in a neighborhood of $P$ such that the restriction of $f(z)$ to an arbitrary analytic plane $\{z \mid z=P+\sigma c\}$ through $P$ is holomorphic. We do this by including in the proof an arbitrary direction $c$ from the beginning.

Proof. Let $f(z)$ be an arbitrary functional $G$-holomorphic in $D$. We consider the subspace $\left\{z \mid z=z_{0}+\lambda b+\tau a+\sigma c\right\}$ where $\sigma$ is a complex parameter and $c$ an arbitrary direction with $\|c\|=1$. The restriction of $f(z)$ to the subspace is a holomorphic function of the three complex variables $\lambda, \tau, \sigma$ (no matter if $a, b, c$ are linearly independent or not.).

For $\lambda \in T$ we have by assumption that $|X(\lambda)| \alpha_{a, D}^{(N)}\left(z_{0}+\lambda b\right) \geq m$, and because $X(\lambda) \neq 0$ on $T$ we have

$$
d_{a, D}^{(N)}\left(z_{\jmath}+\lambda b\right) \geq|X(\lambda)|^{-1} \quad \text { for } \lambda \in T .
$$

Obviously there exists for every $\varepsilon>0$ a sufficiently small $\delta>0$ such that the set

$$
C^{*}=\left\{\left.z\left|z=z_{0}+\lambda b+\tau a+\sigma c,\right| \tau|\leqq(m-\varepsilon)| X(\lambda)\right|^{-1}, \lambda \in T,|\sigma| \leqq \delta\right\}
$$

is contained in $D$ for arbitrary $c$ with $\|c\|=1$. The set $C^{*}$ is compact in the subspace $\left\{z \mid z=z_{\jmath}+\lambda b+\tau a+\sigma c\right\}$, therefore the restriction of $f$ to it is bounded (according to a well known theorem of $n$ complex variables which was first proved by F. Hartogs (compare Carathéodory [9])). Let the bound be $M$. ( $M$ depends upon $c$, of course.)

We now develop the restriction of $f$ in a power series in $\tau$ and $\sigma$.

$$
f\left(z_{0}+\lambda b+\tau a+\sigma c\right)=\left.\sum_{\mu, \nu=0}^{\infty} \frac{1}{\mu ! \nu !} \frac{\partial^{\mu+\nu} f\left(z_{0}+\lambda b+\tau a+\sigma c\right)}{\partial \tau^{\mu} \partial \sigma^{\nu}}\right|_{\tau=\sigma=0} \tau^{\mu} \sigma^{\nu} .
$$

For $\lambda \in T,|\sigma| \leqq \delta$ and $|\tau| \leqq(m-\varepsilon)|X(\lambda)|^{-1}$ the point $z_{0}+\lambda b+\tau a+\sigma c$ belongs to $C^{*}$ where $f$ is holomorphic and its modulus smaller than $M$. Hence we obtain by Cauchy's formula for $\lambda \in T$ the inequality

$$
\left|\frac{1}{\mu ! \nu !} \frac{\partial^{\mu+\nu} f\left(z_{0}+\lambda b+\tau a+\sigma c\right)}{\partial \tau^{\mu} \partial \sigma^{\nu}}\right|_{\tau=\sigma=0} \tau^{\mu} \sigma^{\nu} \mid \leqq \frac{M}{\delta^{\nu}\left[(m-\varepsilon)|X(\lambda)|^{-1}\right]^{\mu}} .
$$

By multiplying with $|X(\lambda)|^{-\mu}$ we obtain for $\lambda \in T$ :

$$
\left.\left|\frac{1}{\mu ! \nu !} \frac{\partial^{\mu+\nu} f\left(z_{0}+\lambda b+\tau \alpha+\sigma c\right)}{\partial \tau^{\mu} \partial \sigma^{\nu}}\right|_{\tau=\sigma=0} \tau^{\mu} \sigma^{\nu}|| X(\lambda)\right|^{-\mu} \leqq \frac{M}{\delta^{\nu}(m-\varepsilon)^{\mu}} .
$$

The left hand side is for $\sigma=\tau=0$ the modulus of a holomorphic function of $\lambda$ and takes its maximum with respect to $S \cup T$ on $T$. Therefore the 
inequality is valid not only for $\lambda \in T$ but for $\lambda \in S \cup T$. Hence the series converges uniformly in every compact subset of the set

$$
C^{* *}=\left\{\left.z\left|z=z_{0}+\lambda b+\tau a+\sigma c, \lambda \in S \cup T,\right| \tau|<(m-\varepsilon)| X(\lambda)\right|^{-1},|\sigma|<\delta\right\} .
$$

The limit function of the series is a continuation of $f\left(z_{0}+\lambda b+\tau a+\sigma c\right)$ into the set $C^{* *}$.

Letting $c$ vary though all directions, that is, through all elements of $B_{c}$ such that $\|c\|=1$ and letting $\varepsilon$ tend to zero we define $f(z)$ in a full neighborhood (in the $B_{c}$ ) of each point of the set

$$
C=\left\{\left.z\left|z=z_{0}+\lambda b+\tau c, \lambda \in S \cup T,\right| \tau|<m| X(\lambda)\right|^{-1}\right\} .
$$

We have to make sure that this definition of $f(z)$ is consistent, that at the same point not two different values are defined!

If $a, b, c_{1}$ and $c_{2}$ are linearly independent, then

$$
z_{1}=z_{0}+\lambda_{1} b+\tau_{1} a+\sigma_{1} c_{1} \neq z_{2}=z_{0}+\lambda_{2} b+\tau_{2} a+\sigma_{2} c_{2}
$$

for all triples

$$
\left(\lambda_{1}, \tau_{1}, \sigma_{1}\right) \neq\left(\lambda_{2}, \tau_{2}, \sigma_{2}\right) .
$$

Therefore no contradictory values can be defined.

Suppose now that $a, b, c_{1}$ and $c_{2}$ are linearly dependent: $c_{2}=\alpha_{1} a+$ $\alpha_{2} b+\alpha_{3} c_{1}$. Then $\lambda_{1}=\lambda_{2}+\sigma_{2} \alpha_{2}, \tau_{1}=\tau_{2}+\sigma_{2} \alpha_{1}$, and $\sigma_{1}=\alpha_{3} \sigma_{2}$ imply $z_{1}=z_{2}$. Let

$$
f\left(z_{0}+\lambda b+\tau a+\sigma c_{1}\right)=f_{1}(\lambda, \tau, \sigma) \text { and } f\left(z_{0}+\lambda b+\tau a+\sigma c_{2}\right)=f_{2}(\lambda, \tau, \sigma) .
$$

Then in a neighborhood of $(0,0,0)$ we have

$$
f_{1}\left(\lambda+\sigma \alpha_{2}, \tau+\sigma \alpha_{1}, \alpha_{3} \sigma\right) \equiv f_{2}(\lambda, \tau, \sigma) .
$$

This functional equation persists wherever both functions are holomorphic (in $\lambda, \tau, \sigma)$. Hence no contradictory values of $f(z)$ are defined at the same point.

Finally we observe that $f$ is by construction $G$-holomorphic in a neighborhood (of the $B_{c}$ ) of each point of $C$. We observe further that $C$ is simply connected. Hence $f$ is single-valued in $C$. Hence the lemma is proved.

3.2. Let the conditions of 3.1 be satisfied except that we replace $d_{a, D}^{(N)}(z)$ by $d_{D}^{(N)}(z)$. Let

$$
|X(\lambda)| d_{D}^{(N)}(z) \geqq m>0 \quad \text { for } \lambda \in T .
$$

Then any functional that is G-holomorphic in $D$ can be continued G-holomorphically into all points 


$$
\tilde{C}=\left\{\left.z\left|\left\|z_{0}+\lambda b-z\right\|<m\right| X(\lambda)\right|^{-1}, \lambda \in S \cup T\right\} .
$$

This follows immediately from the Fundamental Lemma 3.1. For if $|X(\lambda)| d_{D}^{(N)}(z) \geqq m$ for $\lambda \in T$, then in particular $|X(\lambda)| d_{a, D}^{(N)}(z) \geqq m$ for $\lambda \in T$ for every $a$. Hence by Lemma $3.1 f(z)$ is $G$-holomorphically continued into all sets

$$
\left\{\left.z\left|z=z_{0}+\lambda b+\tau a, \lambda \in S \cup T,\right| \tau|<| X(\lambda)\right|^{-1}\right\},
$$

and the union of all these sets is $\tilde{C}$. We observe further that $\tilde{C}$ is simply connected and therefore the continuation single valued.

3.3. How is the continuation of a functional $f, G$-holomorphic in $D$, into a set $\tilde{C}$ as described in 3.2 compatible with values already defined in $D$ ?

If the intersection of $D$ and $\tilde{C}$ is connected, then $f$ is single-valued. Now let $D \cap \tilde{C}$ not be connected. Then there is one component $\tilde{C}_{0}$ of $D \cap \tilde{C}$ containing $S \cup T . \quad f$ is a continuation from $\tilde{C}_{0}$ which can furnish function elements different from the ones already defined in the other components.

We therefore proceed as one does in one and finitely many variables. We pass to "domains over the space." We consider $\tilde{C}$ as the projection of a set $\tilde{C}^{*}$ under a mapping $\varphi$, being a homeomorphism of $\tilde{C}^{*}$ onto $\tilde{C}$. We identify $\tilde{C}_{0}$ and $\tilde{C}_{0}^{*}=\varphi^{-1} \tilde{C}_{0}$, while we consider the other components of $D \cap \tilde{C}$ and their images in $C^{*}$ as different points.

In a further paper we will study the iteration of this process and we will show that in the limit we obtain the simultaneous continuation of all functionals that are holomorphic in $D$ into "the pseudo-convex envelope" of $D$.

3.4. If $D$ is a domain of holomorphy, then the sets $C$ and $\tilde{C}$ belong to $D$. Therefore in this case no question of single-valuedness arises and we can admit $S$ to be an arbitrary domain, not necessarily simply connected.

3.5. CoRollary. Let $D$ be a (schlicht) domain of holomorphy. Let $S$ be an arbitrary domain on an analytic surface $\left\{z \mid z=z_{0}+\lambda b\right\}, T$ the boundary. Then

$$
\inf _{T}|X(\lambda)| d_{a, D}^{(N)}(z)=\inf _{S \cup T}|X(\lambda)| d_{a . D}^{(N)}(z)
$$

and 


$$
\inf _{T}|X(\lambda)| \partial_{D}^{(N)}(z)=\inf _{S \cup T}|X(\lambda)| d_{D}^{(N)}(z)
$$

Let

$$
m=\inf _{T}|X(\lambda)| d_{a, \nu}^{(N)}\left(z_{0}+\lambda b\right) .
$$

Now if $D$ is a domain of holomorphy, then the set $C$ defined in 3.1 belongs to $D$. Hence

$$
d_{a, D}^{(N)}\left(z_{0}+\lambda b\right) \geq m|X(\lambda)|^{-1}
$$

for $\lambda \in S \cup T$. Hence

$$
|X(\lambda)| d_{a, D}^{(N)}\left(z_{0}+\lambda b\right) \geqq m
$$

for $\lambda \in S \cup T$ from which the first equality follows immediately. The second equality follows analogously from Lemma 3.2.

3.6. THEOREM. Let $D$ be a holomorphy, then the functionals $-\log d_{a, D}^{(N)}(z)$ and $-\log d_{D}^{(N)}(z)$ are plurisubharmonic in $D$.

Proof. Suppose $-\log d_{D}^{(N)}(z)$ would not be plurisubharmonic in $D$. Now $-\log d_{D}^{(N)}(z)$ is continuous. Thus there would exist an analytic plane $\left\{z \mid z=z_{0}+\lambda b\right\}$ on which it would not be subharmonic. That means there would exist a (small) circle and a harmonic function $h$ being a majorant on the boundary of the circle but not inside.

We choose the representation of the analytic plane such that $z_{0}$ is the center of the circle. $h(\lambda)$ is harmonic in the open circle $|\lambda|<\rho$ and continuous in $|\lambda| \leq \rho$ and for $|\lambda|=\rho$ we have

$$
-\log d_{D}^{(N)}\left(z_{0}+\lambda b\right) \leq h(\lambda) .
$$

On the other hand there exists a $\lambda_{0}$ with $\left|\lambda_{0}\right|<\rho$ such that

$$
-\log d_{D}^{(N)}\left(z_{0}+\lambda_{0} b\right)>h\left(\lambda_{0}\right) \text {. }
$$

Let $h^{*}(\lambda)$ be a conjugate harmonic function of $h(\lambda)$, then

$$
\left|e^{h(\lambda)+i \iota^{*}(\lambda)}\right| d_{D}^{(N)}\left(z_{0}+\lambda b\right) \geqq 1 \quad \text { for }|\lambda|=\rho
$$

and

$$
\left|e^{h\left(\lambda_{0}\right)+i h^{*}\left(\lambda_{0}\right)}\right| d_{D}^{(v)}\left(z_{0}+\lambda_{0} b\right)<1 \quad \text { for } \lambda_{0} .
$$

This is a contradiction to 3.5 with $T=\{|\lambda|=\rho\}, S=\{|\lambda|<\rho\}$ and $X(\lambda)=$ $e^{h(\lambda)+i l i(\lambda)}$.

Hence $-\log d_{D}^{(N)}(z)$ is plurisubharmonic. The proof for $-\log d_{a, D}^{(N)}(z)$ is analogous. The only difference is that $-\log d_{a, D}^{(N)}(z)$ is upper-semicontinuous instead of continuous. 
4. The Kontinuitätssatz. We will derive now a theorem which in the theory of finitely many variables is known as "Kontinuitätssatz." The term "Kontinuitätssatz" was introduced by Behnke-Thullen [3]; we will use this term because translating it as "theorem of continuity" might be misleading.

4.1. Let $D$ be a domain of holomorphy. Let $\left\{S_{\nu}\right\}$ be a family of bounded domains on one dimensional analytic planes and $\left\{T_{\nu}\right\}$ their boundaries. Let $S_{0}=\lim S_{\nu}$ and $T_{0}=\lim T_{\nu}$. Then $S_{\nu}, T_{\nu} \subset D$ for every $\nu$ and $T_{0} \subset D$ imply $S_{0} \subset D$.

Proof. Applying 3.5 with $X(\lambda) \equiv 1$ we obtain

$$
\inf _{S_{\nu} \cup T_{\nu}} d_{D}^{(N)}(z)=\inf _{T_{\nu}} d_{D}^{(N)}(z)
$$

Now $d_{D}^{(N)}(z)$ is a continuous functional in $D$. Then also $\inf d_{D}^{(N)}(z)$ and $\inf _{T} d_{D}^{(N)}(z)$ are continuous. Therefore the above equality holds also in the limit.

Hence

$$
\inf _{S_{0} \cup P_{0}} d_{D}^{(N)}(z)=\inf _{T_{0}} d_{D}^{(N)}(z)
$$

Now, because $T_{0}$ is compact and in $D$, we have

$$
\inf _{T_{0}} d_{D}^{(N)}(z)>0
$$

and therefore $\inf _{S_{0} \cup T_{0}} d_{D}^{(N)}(z)>0$, which means

$$
S_{0} \subset D
$$

4.2. The Kontinuitätssatz can be expressed also in the following way :

Let $\left\{S_{\nu}\right\}$ be a family of bounded domains on one dimensional analytic planes. Let $S_{0}=\lim S_{\nu}$ and $T_{0}=\lim T_{\nu}$. Let $f(z)$ be a functional holomorphic on $T_{0}$. If then $f(z)$ is singular at least at one point of $S_{0}$, then there exists $a \nu_{0}$ such that for $\nu>\nu_{0}$ the domain $S_{\nu}$ contains at least one singularty of $f(z)$.

5. Pseudo-convex domains. For finite dimensional domains the property of the functional $-\log d_{D}^{(N)}(z)$ to be plurisubharmonic is invariant with respect to the norm (Bremermann [8]). In this section we will extend this result to the infinite dimensional case. As in finite dimension we denote the domains for which the functionals $-\log d_{D}^{(N)}(z)$ are pluri- 
subharmonic as pseudo-convex.

Thus the domains of holomorphy are pseudo-convex. For finite dimension the converse is true: The pseudo-convex domains are domains of holomorphy. This is very deep result due to K. Oka. (K. Oka [14] and [15]). Compare also F. Norgent [13] and H. J. Bremermann [7].) Most of the techniques applied to obtain this result cannot be generalized to infinite dimension (for instance the Weil-Bergmann integral formula [Weil [20], Bergmann [4], [5]] etc.). Nevertheless the pseudo-convexity may be characteristic for domains of holomorphy in the infinite dimensional case also.

5.1. Let $D$ be a domain such that for a certain norm $N$ the functional $-\log d_{a, D}^{(N)}(z)$ is plurisubharmonic in $D$ for every $a$.

Then the intersection $D^{*}$ with any finite dimensional linear submanifold $L$ of $B$ is a pseudo-convex region.

Proof. Let the linear submanifold $L$ be $L=\left\{z \mid z=z_{0}+\tau_{1} b_{1}+\cdots+\tau_{n} b_{n}\right\}$. Let $B^{*}=\left\{z \mid z=\tau_{1} b_{1}+\cdots+\tau_{n} b_{n}\right\}$. The restriction of the norm $N$ to the subspace $B^{*}$ is a norm $N^{*}$ in $B^{*}$. For every $a \in B^{*}$ the restriction of $d_{a, D}^{(N)}(z)$ to $D^{*}=L \cap D$ is equal to $d_{a, D *}^{(N)}(z)$ by definition.

The restriction of any plurisubharmonic functional in $D$ to $D^{*}$ is a plurisubharmonic function in $D^{*}$, as one sees immediately from the definition of the plurisubharmonic functions.

Hence $-\log d_{a, D *}^{(N *)}(z)$ is a plurisubharmonic function for every $a \in B^{*}$. Hence (the finite dimensional) $D^{*}$ is pseudo-convex according to a result by Bremermann [8].

REMARK. The intersection $D \cap L$ is not necessarily connected, but just an open set, that is, a region.

5.2. Let $D$ be a domain such that the intersection $D^{*}$ of $D$ with any two dimensional linear submanifold $L$ of $B$ is pseudo-convex, then $-\log d_{a_{1} D}^{(N)}(z)$ is plurisubharmonic in $D$ for every a and for any norm $N$, which generates a topology which is equivalent to the topology with respect to which $D$ is defined.

Proof. For finite dimensional Banach spaces it has been proved in Bremermann [8] that if $D^{*}$ is a pseudo-convex region then $-\log d_{a, D^{*}}^{\left(N^{*}\right)}$ is plurisubharmonic in $D^{*}$ for any norm $N^{*}$. Now if $N$ is an arbitrary norm, then its restriction $N^{*}$ to a finite dimensional subspace $B^{*}$ is a norm in $B^{*}$. Hence $-\log d_{a, D^{*}}^{\left(N^{*}\right)}(z)$ is plurisubharmonic in $D^{*}$ for $a \in B^{*}$ for every two dimensional subspace $B^{*}$, hence the restriction of $-\log d_{D}^{(N)}(z)$ to any $D^{*}$ is plurisubharmonic, hence $-\log d_{a, D}^{(N)}(z)$ is pluri- 
subharmonic in $D$.

\section{1 and 5.2 combined yield:}

5.3. COROLLARY. The property of the functionals $-\log d_{a, D}^{(N)}(z)$ to be plurisubharmonic for a domain $D$ is invariant with respect to all norms that generate equivalent topologies.

5.4. Definition. The domains which have the property that the functionals $-\log d_{a, D}^{(N)}(z)$ are plurisubharmonic for all $a$ we call pseudoconvex.

5.5. COROLlary. The domains of holomorphy are pseudo-convex.

5.6. With this definition we can express 5.1 and 5.2 also in the following way.

$A$ domain $D$ is pseudo-convex if and only if the intersection $D$ with any finite dimensional linear submanifold is a pseudo-convex region.

The same is true if we replace "finite dimensional" by "two dimensional."

5.7. We now replace the functionals $-\log d_{a, D}^{(N)}(z)$ by $-\log d_{D}^{(N)}(z)$ and show: $D$ is pseudo-convex if and if $-\log d_{D}^{(N)}(z)$ is plurisubharmonic for arbitrary norms $N$ with equivalent topology.

Let $D^{*}$ be as in 5.1. If $-\log d_{D}^{(N)}(z)$ is plurisubharmonic, then its restriction to $D^{*}$ is plurisubharmonic. In general, however, we cannot say that this restriction is equal to $-\log d_{D^{*}}^{(N)}(z)$.

Let the finite dimensional submanifold be

$$
\left\{z \mid z=z^{0}+\tau_{1} b_{1}+\cdots+\tau_{n} b_{n}\right\} .
$$

Then we take the upper envelope of

$$
\left\{-\log d_{D}^{(N)}(z), \log \left|\tau_{1}\right|, \cdots \log \left|\tau_{n}\right|\right\} .
$$

This is a plurisubharmonic function that becomes infinite everwhere at the boundary of $D^{*}$. Hence the finite dimensional region $D^{*}$ is pseudoconvex according to Bremermann [8]. Hence $D$ is pseudo-convex according to 5.6.

On the other hand if $D$ is pseudo-convex, then

$$
-\log d_{a, D}^{(N)}(z)
$$

is plurisubharmonic for every $a$ by definition. Now we have 


$$
-\log d_{D}^{(N)}(z)=\sup _{a}\left\{-\log d_{a, D}^{(N)}(z)\right\} .
$$

In particular this relation holds also on every analytic plane. On analytic planes $-\log d_{J)}^{(N)}(z)$ is thus the upper envelope of subharmonic functions, and thus according to Radó [16], subharmonic. Hence $-\log d_{I}^{(N)}(z)$ is plurisubharmonic in $D$.

5.8. A domain $D$ is pseudo-convex if there exists a plurisubharmonic functional $V(z)$ such that the closure of

$$
\{z \mid V(z)<M, z \in D\}
$$

is contained in $D$ for arbitrary large $M$. If $D$ is bounded, then the converse is true.

Proof. If there exists such a $V(z)$, then we restrict $V(z)$ to finite dimensional subspaces and obtain by the analog theorem from the finite dimensional case (Bremermann [8] that all $D^{*}$ are pseudo-convex, hence $D$ is pseudo-convex.

On the other hand, if $D$ is pseudo-convex, then $-\log _{D}^{(N)}(z)$ is plurisubharmonic and will tend to infinity at any finite boundary point of $D$.

5.9. Let $D$ be a pseudo-convex domain. Then the Kontinuitätssatz holds for D. (Compare 4.1).

Proof. If $D$ is pseudo-convex, then $-\log d_{D}^{(N)}(z)$ is plurisubharmonic (5.7). Then the restriction of $-\log d_{D}^{(N)}(z)$ to a one dimensional analytic plane is subharmonic. For subharmonic functions the maximum principle holds (Radó [16]). Hence we have

$$
\inf _{S_{\nu} \cup T_{\nu}} d_{D}^{(N)}(z)=\inf _{T_{\nu}} d_{D}^{(N)}(z) \quad \text { for every } \nu .
$$

The rest of the proof follows as in 4.1.

5.10. Most theorems which hold for plurisubharmonic functions and pseudo-convex domains in the finite dimensional case also hold in the infinite dimensional case. (For instance: The intersection of two pseudo-convex domains is a pseudo-convex region.) We have listed here only some of the very basic facts. The reader will find it not difficult to extend most of the theorems listed in Complex convexity (Bremermann [8]) to the infinite dimensional case.

In [8] we have stressed the formal relationship between complex convexity (by which notion we denote the plurisubharmonic functions and the pseudo-convex domains jointly) to ordinary convexity.

In the following section we will show that the same relationship 
persists in the infinite dimensional case.

\section{Elementary convexity and its relation to complex convexity.}

6.1. A real valued function $U(t)$ of one real variable $t$ is called convex in an interval $D$ of the real $t$-axis if and only if the following condition holds for every closed subinterval $D^{\prime} \subset D$ :

If $l(t)$ is a linear function such that $l(t) \geqq U(t)$ on the boundary of $D^{\prime}$, then $l(t) \geqq U(t)$ holds also for $t \subset D^{\prime}$.

6.2. A real valued functional $U(x)$ defined in a domain $D$ of a real Banach space $B_{r}$ is called convex if and only if its restriction to an arbitrary straight line $\left\{x \mid x=x_{0}+t a\right\}$ is a convex function of $t$ in $\{x \mid x=$ $\left.x_{0}+t a\right\} \cap D$.

REMARK. Formally these definitions are similar to the definitions of subharmonic and plurisubharmonic functionals (compare Bremermann [8]).

6.3. A domain $D$ in a real Banach space $B_{r}$ is convex if with any two points $x_{1}$ and $x_{2}$ the connecting straight line segment $\left\{x \mid x=x_{1}+\right.$ $\left.t\left(x_{2}-x_{1}\right), 0 \leqq t \leqq 1\right\}$ is contained in $D$.

REMARK. This definition bears no formal relationship to the definition of the pseudo-convex domains. We will establish this relationship-as in the finite dimensional case (compare Bremermann [8])-by proving that a domain $D$ is convex if and only if $-\log d_{D}^{(N)}(x)$ is a convex functional in $D$. The proof which we have given in [8] for finite dimension does not apply for infinite dimension, therefore a different one is given in the following. (Convexity in several complex variables has also been studied from a different point of view by Behnke-Stein [2].)

6.4. Let $D$ be a convex domain. Let $S$ be an interval on a straight line $\left\{x \mid x=x_{0}+t b\right\}, T$ the boundary of $S$. Let $l(t)$ be a linear function of $t$. Then we have for every $a \in B,\|a\|=1$

$$
\inf _{T} d_{a, D}^{(N)}\left(x_{0}+t b\right) e^{l(t)}=\inf _{S \cup T} d_{a, D}^{(N)}\left(x_{0}+t b\right) e^{l(t)} .
$$

Proof. In the following we consider the subspace generated by the vectors $a$ and $b$. Let the parameter values belonging to the two points of $T$ be $t_{1}$ and $t_{2}\left(t_{1}<t_{2}\right)$.

We observe that all the points

$$
x=x_{0}+t_{1} b+\vartheta d_{a_{1} D}^{(N)}\left(x_{0}+t_{1} b\right) \cdot a
$$


and

$$
x=x_{0}+t_{2} b+\vartheta d_{a . D}^{(N)}\left(x_{0}+t_{2} b\right) \cdot a
$$

belong to $D$ for $-1<\vartheta<1$ by definition of $d_{a, D}^{(N)}(x)$. Now $D$ is convex by assumption. Hence all the points on the connecting straight line segment connecting any two of these points belong to $D$. Now let $\inf _{T} d\left(x_{0}+t b\right) e^{l(t)}=m>0$. Then

$$
\left.d\left(x_{0}+t_{1} b\right) e^{l(t)}\right)_{1} \geqq m \quad \text { and } \quad d\left(x_{0}+t_{2} b\right) e^{l(t)_{2}} \geqq m .
$$

Hence all the points on the straight line segment passing through

$$
x_{1}(\vartheta)=x_{0}+t_{1} b+\vartheta m e^{-l\left(t_{1}\right) a} \quad \text { and } \quad x_{2}(\vartheta)=x_{0}+t_{2} b+\vartheta m e^{-l\left(t_{2}\right)} a
$$

belong to $D$ for $-1<\vartheta<1$.

Now the function $m e^{-l(t)}$ is a convex function of $t$ for any linear function $l(t)$. Hence the curve

$$
x\left(t_{1}, \vartheta\right)=x_{0}+t b+\vartheta m e^{-l(t)} a, \quad t_{1} \leqq t \leqq t_{2}
$$

will lie for $-1<\vartheta<1$ within the parallelogram through the four points $x_{1}(1), x_{1}(-1), x_{2}(1), x_{2}(-1)$. Hence

$$
d_{a, D}^{(N)}\left(x_{0}+t b\right) \geqq m e^{-l(t)} \quad \text { for } \quad t_{1} \leqq t \leqq t_{2} .
$$

And therefore

$$
d_{a, D}^{(N)}\left(x_{0}+t b\right) e^{l(t)} \geq m
$$

and

$$
\inf _{S \cup T} d_{a, D}^{(N)}\left(x_{0}+t b\right) e^{l(t)} \geqq m
$$

6.5. Let $D$ be a domain such that for an arbitrary linear function $l(t)$ and line segments $s$ with boundary $T$ we have

$$
\inf _{T} d_{a, D}^{(N)}(x) e^{l(t)}=\inf _{S \cup T} d_{a, D}^{(N)}(x) e^{l(t)} .
$$

Then $-\log d_{a, D}^{(N)}(x)$ is a convex function in $D$.

Proof. Suppose $-\log d_{a, D}^{(N)}(x)$ would not be convex. Then there would exist a straight line $\left\{x \mid x=x_{0}+t b\right\}$ and a segment $S$ with boundary $T$ and a linear function $l(t)$, such that

$$
l(t) \geqq-\log d_{a, D}^{(N)}\left(x_{0}+t b\right)
$$

on $T$, but there would exist a $t_{0} \in S$ such that

$$
l\left(t_{0}\right) \leqq-\log d_{a, D}^{(N)}\left(x_{0}+t_{0} b\right) .
$$


This is equivalent to

$$
1 \leqq d_{a . D}^{(N)}\left(x_{0}+t b\right) e^{\tau(t)}
$$

on $T$, and

$$
1>d_{a, D}^{(N)}\left(x_{0}+t_{0} b\right) e^{l\left(t_{0}\right)} .
$$

Thus the minimum principle would be violated in contradiction to the assumption, hence the functional $-\log d_{a, D}^{(N)}(x)$ is convex in $D$.

6.6. Summing up 6.4 and 6.5: If $D$ is a convex domain, then for an arbitrary norm $N$ (generating an equivalent topology) and for every $a \in B_{r}$ with $\|a\|=1$ the functional $-\log d_{a, D}^{(N)}(x)$ is convex in $D$.

6.7. If $D$ is convex, then $-\log d_{D}^{(N)}(x)$ is convex in $D$ for an arbitrary norm $N$ (with equivalent topology).

This is an immediate consequence of 6.6 because $-\log d_{D}^{(N)}(x)$ is the upper envelope of the family $\left\{-\log d_{a, D}^{(N)}(x)\right\}$ and the upper envelope of a locally upper bounded family of convex functionals is convex.

6.8. We now proceed to prove the converse of 6.7 , and for this purpose we show first:

Let $D$ be a domain such that for one particular norm the functional $-\log d_{D}^{(N)}(x)$ is convex. Then the following "Kontinuitätssatz" holds for $D$ :

Let $\left\{S_{\nu}\right\}$ be a family of straight line segment, $T_{\nu}$ their boundaries and $S_{0}=\lim _{\nu \rightarrow \nu_{0}} S_{\nu}, T_{0}=\lim _{\nu \rightarrow \nu_{0}} T_{\nu}$. Then $\left.\left\{S_{\nu}\right\}, T_{\nu}\right\}, T_{0} \subset D$ implies $S_{0} \subset D$.

The proof is analogous to the proof of 5.9 and 4.1.

6.9. If for a domain $D$ the Kontinuitätssatz 6.8 holds, then $D$ is convex.

We have to show: Let $x_{1}, x_{2}$ be two arbitrary points in $D$. Then we can connect them by a straight line segment.

Since $D$ is a domain, we can connect $x_{1}$ and $x_{2}$ by a continuous arc $x(t)$. Let $x(0)=x_{1}$ and $x(1)=x_{2}$. We connect $x_{1}$ with $x(t)$ by the straight line segment. For small $t$ the point $x(t)$ is in a neighborhood of $x_{1}$ and therefore the connecting line segment in $D$. Now, there cannot be a first line segment $\left\{x_{1}, x\left(t_{1}\right)\right\}$ such that for $0 \leqq t<t_{1}$ the line segments are in $D$, however $\left\{x_{1}, x\left(t_{1}\right)\right\}$ is not, because this would violate the Kontinuitätssatz. Hence the line segment connecting $x_{1}$ and $x_{2}$ is in $D$, hence $D$ is convex.

6.10. Summing up the results of this section:

$A$ domain $D$ is convex if and only if 
(a) $-\log d_{D}^{(N)}(x)$ is convex in $D$. This property is invariant with respect to all topologically equivalent norms.

(b) $-\log d_{a, D}^{(N)}(x)$ is convex in $D$ for all $a \in B_{r}$ with $\|a\|=1$. This property, too, is invariant with respect to all topologically equivalent norms.

(c) The Kontinuitätssatz 6.8 holds for $D$.

6.11. We add : $D$ is convex if and only if the intersection of $D$ with any finite dimensional (two dimensional) linear submanifold of $B_{r}$ is convex. This is obvious. We note further that most of the theorems and analogies to complex convexity which for the finite dimensional case are explicated in [8] are true for the infinite dimensional case also. The reader will find it very easy to carry out the proofs himself.

\section{Tube domains.}

7.1. Let $B_{r}$ be a real Banach space. Then we can define a complex Banach space by considering pairs of elements of $B_{r}$ :

$$
B_{c}=\{x, y\}, \quad x \in B_{r}, \quad y \in B_{r},
$$

and by defining for complex scalars $\lambda=\sigma+i \tau$ the multiplication

$$
\lambda(x, y)=(\sigma x-\tau y, \sigma y+\tau x) .
$$

As usual, we will write $(x, y)=x+i y$. If \|\|$_{r}$ is the norm defined in $B_{r}$, then one defines

$$
\|x+i y\|_{c}=\left(\|x\|_{r}^{2}+\|y\|_{r}^{2}\right)^{1 / 2}
$$

and one easily checks that the axioms are satisfied.

7.2. Definition. Let $B_{c}$ be a complex vector space and $B_{x}$ and $B_{y}$ its "real" and "imaginary" components. Then a tube domain is a domain that has the form

$$
T_{X}=\left\{z \mid z=x+i y, x \in X, y \in B_{y}\right\},
$$

where $X$ is a domain in $B_{x}$ called the basis of $T_{X}$. (The notion of tube domain was introduced in the finite dimensional case by Bochner-Martin [6].)

7.3. Any functional $f(z)$ holomorphic in a tube domain $T_{X}$ is determined throughout $T_{X}$ already by its values in the basis $X$ of $T_{X}$.

Proof. Let us consider the analytic plane passing through the two points 


$$
z_{1}=x_{0} \quad \text { and } \quad z_{2}=x_{0}+i y_{0}, \quad x_{0} \in X, \quad y_{0} \text { arbitrary }
$$

The parameter representation is

$$
z=z_{1}+\lambda^{\prime}\left(z_{2}-z_{1}\right)=x_{0}+i \lambda^{\prime} y_{0}=x_{0}+\lambda y_{0} .
$$

Its intersection with the $B_{x}$ is

$$
z=x_{0}+(\mathscr{R} \lambda) y_{0} \text {. }
$$

This is a straight line passing through $x_{0}$. On the intersection of this straight line with $X$ the values of $f(z)$ are prescribed. Then $f(z)$ is determined on the whole plane strip

$$
\left\{z \mid z=x_{0}+\lambda y_{0}, \mathscr{R}\left(x_{0}+\lambda y_{0}\right) \in X\right\}
$$

by a classical theorem on functions of one complex variable.

The union of the analytic planes considered contains the whole tube $T_{X}$, hence we conclude that $f(z)$ is determined throughout $T_{X}$.

7.4. An upper semi-continuous functional $V(z)$ defined in a tube domain $T_{X}$ that does not depend upon the imaginary part of $z$ is a plurisubharmonic functional in $T_{X}$ if and only if its restriction to the basis $X$ is a convex functional in $X$.

Proof. Let $V(z)$ be plurisubharmonic. Then $V(z)$ is subharmonic on every analytic plane $\left\{z \mid z=z_{0}+\lambda a\right\}$. Then the Laplacian

$$
\left.\frac{\partial^{2} V\left(z_{0}+\lambda a\right)}{\partial \lambda \partial \bar{\lambda}}\right|_{\lambda=0} \geq 0
$$

for every $a \in B_{c}$ and $z_{0} \in T_{x}$. (Taken in the sense of L. Schwartz.)

Let $\lambda=\sigma+i \tau$ and $a=c+i d$. Then

$$
z_{0}+\lambda a=x_{0}+i y_{0}+\sigma c-\tau d+i(\tau c+\sigma d) .
$$

Now if $V(z)$ does not depend upon $y$, then

$$
V\left(z_{3}+\lambda a\right)=V\left(x_{0}+\sigma c-\tau d\right) .
$$

Hence

$$
\left.\frac{\partial^{2} V\left(z_{0}+\lambda a\right)}{\partial \lambda \partial \bar{\lambda}}\right|_{\lambda=0}=\left.\left(\frac{\partial^{2}}{\partial \sigma^{2}}+\frac{\partial^{2}}{\partial \tau^{2}}\right) V\left(x_{0}+\sigma c-\tau d\right)\right|_{\sigma=\tau \approx 0}
$$

For $d=0$ it follows that

$$
\left.\frac{\partial^{2} V\left(x_{0}-\sigma c\right)}{\partial \sigma^{2}}\right|_{\sigma=0} \geqq 0
$$


and letting $c=0$ we obtain

$$
\left.\frac{\partial^{2} V\left(x_{0}-\tau d\right)}{\partial \tau^{2}}\right|_{\tau=0} \geqq 0
$$

This means however that $V(x)$ is a convex function in $X$.

On the other hand, if $V(x)$ is convex, then

$$
\left.\frac{\partial^{2} V\left(x_{0}+\sigma c\right)}{\partial \sigma^{2}}\right|_{\sigma=0} \geqq 0 \quad \text { and }\left.\quad \frac{\partial^{2} V\left(x_{0}-\tau d\right)}{\partial \tau^{2}}\right|_{\tau=0} \geqq 0
$$

for every $c, d \in B_{r}, x_{0} \in X$, and therefore

$$
\left.\frac{\partial^{2} V\left(z_{0}+\lambda a\right)}{\partial \lambda \partial \bar{\lambda}}\right|_{\lambda=0} \geqq 0
$$

for every $a \in B_{c}$ and $z_{0} \in T_{x}$. Hence $V(z)$ is subharmonic on every analytic plane. $V(z)$ is by assumption upper semi-continuous. Hence $V(z)$ is plurisubharmonic.

7.5. A tube domain $T_{X}$ is pseudo-convex if and only if its basis $X$ is convex.

Corollary. A tube domain is pseudo-convex if and only if it is convex.

Let $T_{X}$ be pseudo-convex. Then $-\log d_{p_{X}}^{(N)}(z)$ is plurisubharmonic and it does not depend upon the imaginary part $y$. Then norm $N$ generates a norm $N^{\prime}$ in $B_{x}$, and the restriction of $-\log d_{T_{X}}^{(N)}(z)$ to $X$ is equal to $-\log d_{X}^{\left(N^{\prime}\right)}(x)$. Hence $-\log d_{X}^{\left(N^{\prime}\right)}(x)$ is convex, hence the domain $X$ is convex by Theorem 6.10. And passing through the conclusions in the reverse direction we conclude conversely if $X$ is convex, then $T_{X}$ is pseudo-convex.

Obviously $T_{X}$ is convex if and only if $X$ is convex. Hence follows the corollary.

7.6. It can be shown that all holomorphic functionals can be continued into the "pseudo-convex envelope." In the case of our tube domain $T_{X}$ the pseudo-convex envelope is that tube that has the convex envelope of $X$ as its base.

This, however, we will study in a further paper.

\section{REFERENCES}

1. H. Behnke and F. Sommer, Über die Voraussetzungen des Kontinuitätssatzes, Math. Ann., 121 (1950), 356-378. 
2. H. Behnke and K. Stein, Die Konvexität in der Funktionentheorie mehrerer komplexer Veränderlichen, Mitt. Math. Ges. Hamburg, 8 (1940).

3. H. Behnke and P. Thullen, Theorie der Funktionen mehrerer komplexer Veränderlichen, Ergebn. d. Math., 3, no. 3 (1934).

4. S. Bergman, $\ddot{U} b e r$ eine in gewissen Bereichen gültige Integraldarstellung von Funktionen zweier komplexer Variabler, Math., 39 (1934), 76-94 and 605-608.

5. - - Über eine Integraldarstellung von Funktionen aweier komplexer Veränderlicher, Rec. Math. (Mat.), N.S. 1 (43), (1936), 851-862.

6. $\quad$ and W. T. Martin, Several complex variables, Princeton 1948.

7. H. J. Bremermann, Über die Äquivalenz der pseudokonvexen Gebiete und der Holomorphiegebiete im Raum von $n$ komplexen Veränderlichen Math. Ann., 128 (1954), 63-91.

8. - Complex convexity, to appear in Trans. Amer. Math. Soc.

9. C. Carathéodory Funktionentheorie, vol. 2 Basel 1950.

10. J. Eells, The differential geometry of mapping spaces. To appear.

11. E. Hille, Functional analysis and semi-groups, New York 1948.

12. P. Lelong, Les fonctions plurisosharmoriques, Ann. Sci. Ecole. Norm. Sup., 62 (1945), 301-338.

13. F. Norguet, Sur les domaines d'holomorphie des fonctions uniformes de plusieur variables complexes, Bull. Soc. Math. France, 82 (1954), 137-159.

14. K. Oka, VI, Domaines pseudoconvexes, Tôhoku Math. J., 49 (1942), 15-52.

15. - IX, Domaines finis sans point critique intérieur, Jap. J. Math., 23 (1953), 97-155.

16. T. Radó, Subharmonic functions, Ergebn. d. Math., 5, no. 1 (1937).

17. H. Soeder, Funktionentheorie in Banachschen Räumen, Staatsarbeit at the Math. Inst. Univ. Münster, 1954; Thesis Münster 1956.

18. A. E. Taylor, Analysis in complex Banach spaces, Bull. Amer. Math. Soc., 49 (1943), 652-669.

19. G. O. Thorin, Convexity theorems, Medd. Lunds Univ. Mat. Sem., 9 (1948).

20. A. Weil, L'intégrale de Cauchy et les fonctions de plusieur variables, Math. Ann., 111 (1935), 178-182. 



\section{PACIFIC JOURNAL OF MATHEMATICS}

EDITORS

H. L. ROYDEN

Stanford University

Stanford, California

R. A. Beaumont

University of Washington

Seattle 5 , Washington
A. R. Whiteman

University of Southern California

Los Angeles 7, California

E. G. Straus

University of California

Los Angeles 24, California

\section{ASSOCIATE EDITORS}
E. F. BECKENBACH
C. E. BURGESS
M. HALL
E. HEWITT
A. HORN
V. GANAPATHY IYER
R. D. JAMES
M. S. KNEBELMAN

L. NACHBIN

I. NIVEN

G. SZEKERES

T. G. OSTROM

M. M. SCHIFFER
F. WOLF

K. YOSIDA

\section{SUPPORTING INSTITUTIONS}

UNIVERSITY OF BRITISH COLUMBIA

CALIFORNIA INSTITUTE OF TECHNOLOGY

UNIVERSITY OF CALIFORNIA

MONTANA STATE UNIVERSITY

UNIVERSITY OF NEVADA

OREGON STATE COLLEGE

UNIVERSITY OF OREGON

UNIVERSITY OF SOUTHERN CALIFORNIA

\author{
STANFORD UNIVERSITY \\ UNIVERSITY OF UTAH \\ WASHINGTON STATE COLLEGE \\ UNIVERSITY OF WASHINGTON \\ AMERICAN MATHEMATICAL SOCIETY \\ CALIFORNIA RESEARCH CORPORATION \\ HUGHES AIRCRAFT COMPANY \\ THE RAMO-WOOLDRIDGE CORPORATION
}




\section{Pacific Journal of Mathematics}

\section{Vol. 7, No. $1 \quad$ January, 1957}

Richard Davis Anderson, Zero-dimensional compact groups of

homeomorphisms ................................... 797

Hans-Joachim Bremermann, Holomorphic functionals and complex

convexity in Banach spaces........................... 811

Hugh D. Brunk, G. M. Ewing and W. R. Utz, Minimizing integrals in

certain classes of monotone functions ................. 833

Philip David, Uniqueness theory for asymptotic expansions in general

regions ...................................... 849

Paul Erdős and Harold Nathaniel Shapiro, On the least primitive root of a

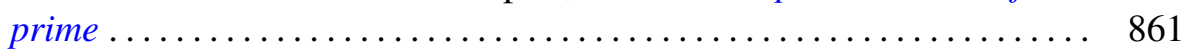

Watson Bryan Fulks, Regular regions for the heat equation ........... 867

William Robert Gaffey, A real inversion formula for a class of bilateral

Laplace transforms ................................ 879

Ronald Kay Getoor, On characteristic functions of Banach space valued random variables ................................. 885

Louis Guttman, Some inequalities between latent roots and minimax (maximin) elements of real matrices ...................... 897

Frank Harary, The number of dissimilar supergraphs of a linear graph .... 903

Edwin Hewitt and Herbert S. Zuckerman, Structure theory for a class of convolution algebras .................................. 913

Amnon Jakimovski, Some Tauberian theorems . . . . . . . . . . . . . . . . . 943

C. T. Rajagopal, Simplified proofs of "Some Tauberian theorems" of Jakimovski................................

Paul Joseph Kelly, A congruence theorem for trees ................. 961

Robert Forbes McNaughton, Jr., On the measure of normal formulas...... 969

Richard Scott Pierce, Distributivity in Boolean algebras .............. 983

Calvin R. Putnam, Continuous spectra and unitary equivalence ......... 993

Marvin Rosenblum, Perturbation of the continuous spectrum and unitary

equivalence................................... 997

V. N. Singh, Certain generalized hypergeometric identities of the

Rogers-Ramanujan type.......................

Peter Swerling, Families of transformations in the function spaces $H^{p} \ldots \ldots 1015$ 